schichtet, dann das offene Ende der Röhre capillarisch ausgezogen und zugeschmolzen. Beim mässigen Erhitzen, welches die zum Austreiben des Quecksilbers nothwendige Temperatur nicht überschritten hatte, wurde ein metallischer Spiegel erhalten, welcher vom Quecksilberbeschlag äusserlich kaum zu unterscheiden war. Bei stärkerem Glïhen wurden am Anfang der Capillare glänzende Kügelchen beobachtet, welche den Quecksilbertröpfchen sehr ähnlich aussahen, beim Antasten aber sich als harte Körnchen des Cadmiummetalles erwiesen. In dem angewandten Zinkpulver wurde der Cadmiumgehalt zu 1,63\% gefunden. Durch starkes Glühen wird dasselbe gar nicht vollständig entfernt, im Gegentheil verflüchtigt sich das Cadmium nur langsam. Nach 10 Minuten langem Glühen im bedeckten Porzellantiegel wurde der Cadmiumgehalt noch zu 1,50\% gefunden. Dasselbe Zinkpulver wurde auch 1 Stunde lang in einem Rohr im Wasserstoffstrom erhitzt; sein Cadmiumgehalt betrug alsdann doch noch $1 \%$.

Um also den möglichen Fehler zu vermeiden, darf bei Anwendung des Zinkpulvers die Controlprüfung des erhaltenen Metallspiegels mit Jod niemals unterlassen werden. Wir möchten aber auch den weniger Geübten entgegenkommen und statı Zinks das von E. Ludwig selbst vorgeschlagene Kupferpulver oder das später von anderen angewandte Kapferblech oder Kupferspäne empfehlen.

Ueber eine neue Methode zum. Nachweis von Baumwollsamenöl in Schweinefett und Olivenöl und über die annähernde Schätznng des Gehalts an Baumwollsamenöl im Schweinefett.

$$
\text { Yon }
$$

\title{
F. Gantter.
}

Bei der Prüfung zahlreicher Proben von Schweinefett nach der Methode von Becchi war es mir aufgefallen, dass bei keiner einzigen die Schwärzung des Silbernitrats eintrat, trotzdem bei verschiedenen dieser Proben nach Preis und anderen Anzeichen fast mit Sicherheit anzunehmen war, dass sie mit Cotton- oder einem anderen Oele vermischt waren. Ich prüfte nun in gleicher Weise 3 Proben Cottonöl, von 3 verschiedenen Quellen bezogen, ohne jedoch auch hier das Eintreten der Reaction beobachten zu können. Es scheint also jetzt Baumwollsamenöl in den Handel zu 
kommen, welches in Folge Erhitzens oder anderer Behandlung die Reaction mit Silbernitrat nicht mehr zeigt. Es ist daher wahrscheinlich, dass die Reduction des Silbernitrats, nicht wie Becchi ${ }^{1}$ ) ursprünglich annahm, durch das Glykosid des Baumwollsamenöls verursacht wird, sondern vielmehr durch unbestimmte Verunreinigungen, welche durch geeignete Behandlung entfernt werden können. Man wird also wohl annehmen können, dass die zur Erkennung von Baumwollsamenöl vorgeschlagenen Reductionsmethoden jedenfalls nicht in allen Fällen den sicheren Nachweis dieses Oeles gestatten. Ich sah mich daher veranlasst, nach einer anderen Methode zum Nachweis dieses Oeles im Schweinefett zu suchen. $\mathrm{Zu}$ den ältesten und am häufigsten angewandten Reagentien zur Prüfung der Fette gehört die concentrirte Schwefelsäure. Schweine- and Butterfett, Oliven- und andere Oele werden durch concentrirte Schwefelsäure sehr stark angegriffen, so dass, wenn man letztere direct auf Fett oder Oel einwirken lässt, die Reaction leicht $z u$ weit geht und dann in einer Weise verläuft, welche zwischen den einzelnen Oelen und Fetten einen deutlichen, stets sicher erkennbaren Unterschied nicht wahrnehmen lässt. Die Anwendung verdünnter Schwefelsäure ist aber ausgeschlossen, da diese auf die Fette und Oele nicht genügend einwirkt. Auf diesen Umstand hat bereits F. A. Flü ckiger ${ }^{2}$ ) aufmerksam gemacht und vorgeschlagen, bei der Prüfung die concentrirte Schwefelsäure auf die durch Aether, Benzol, Chloroform, Schwefelkohlenstoff etc. verdünnten Oele einwirken zu lassen. Die concentrirte Schwefelsäure wirkt in der That auf die durch die genannten Lösungsmittel verdünnten Fette und Oele noch sehr energisch ein, so dass selbst hier, bei Anwendung zu grosser Schwefelsäuremengen, der Verlauf der Reaction so weit geht, dass ein Unterschied zwischen verschiedenen Oelen nicht mehr mit Sicherheit zu erkennen ist. Lässt man aber sehr wenig concentrirte Schwefelsäure auf verhältnissmässig viel durch das Lösungsmittel verdünntes Oel einwirken, so wird dadurch die Reaction bedeutend gemässigt und lassen sich die dabei auftretenden Erscheinungen leicht beobachten. Wie ich durch verschiedene Versuche gefunden habe, gelingt dies am schärfsten, wenn man so verfährt: In ein Reagirglas bringt man 1 cc geschmolzenes Fett oder Oel, welche vollkommen wasserfrei sein müssen, löst in $10 \mathrm{cc}$ Petroleumäther, lässt, am besten aus einem Tropfglas, einen Tropfen con-

1) Vergl. diese Zeitschrift $\mathbf{2 3}, 97$.

2) Diese Zeitschrift 10, 235. 
centrirte Schwefelsäure in die Lösung fallen und schüttelt sofort stark um. Die bei dieser Art der Ausführung der Reaction auftretenden Farbenerscheinungen sind sehr deutlich und lassen Unterschiede in dem Verhalten verschiedener Fette gut erkennen.

Ich habe zunächst das Verhalten von Cottonöl und Schweinefett bei dieser Behandlung beobachtet und dabei einen wesentlichen Unterschied zwischen beiden gefunden. Reines Schweinefett färbt sich nämlich bei dieser Behandlung hell strohgelb bis schwach röthlichgelb; die Lösung bleibt anfangs klar, nach und nach setzen sich aus derselben stark rothgelb gefärbte schwere Tropfen $\mathrm{ab}$, während die darüber stehende Flüssigkeit kaum gelblich gefärbt, in den meisten Fällen wasserhell bleibt. Cottonöl dagegen färbt sich sofort stark braun bis schwarz und behält diese Farbe auch nach sehr langem Stehen. Gemenge von Schweinefett und Cottonöl färben sich, je nach dem Gehalt an letzterem, mehr oder weniger dunkelbraun. Diese dunkelbraune Färbung tritt selbst bei einem Gehalt von nur 1\% Cottonöl im Schweinefett noch deutlich auf.

Olivenöl zeigt bei dieser Behandlung eine ganz ähnliche Erscheinung wie Schweinefett. Reines Olivenöl färbt sich nach dem $\mathrm{Zu}$ satz von 1 Tropfen concentrirter Schwefelsäure schwach dunkel; nach zweistündigem Stehen setzt sich ein stark rothgelb gefärbtes, dickes oel zu Boden und die darüber stehende Flüssigkeit ist beinahe wasserhell. Enthält das Olivenöl aber Cottonöl, so färbt es sich sofort dunkelbraun bis schwarzbraun, und diese Färbung bleibt auch nach sehr langem Stehen. Erdnussöl verhält sich ähnlich wie Cottonöl, nur tritt keine so starke Färbung auf wie bei letzterem. Olivenöl, welches bei dieser Behandlung eine auch nach langer Zeit dunkel gefärbt bleibende Lösung ergibt, kann daher Cotton- oder Erdnussöl enthalten.

Wie im Vorstehenden ausgeführt wurde, kann bei der Prüfung von Schweinefett aus dem Nichteintreten der Silbernitratprobe ein sicherer Schluss auf die Abwesenheit von Cottonöl nicht gezogen werden. Das Eintreten der Reaction kann zwar zum qualitativen Nachweise dienen, gestattet aber nicht die Menge des Cottonöls festzustellen. Wohl hat man versucht aus der Intensität der eintretenden Färbung die Menge des Cottonöls zu schätzen; da aber die Intensität der Färbung, wenn sie überhaupt eintritt, von verschiedenen Nebenumständen abhängt, so kann ein derartiger Schluss nicht als berechtigt erachtet werden. Eine 
wesentlich genauere und auf controlirbarer Grundlage beruhende Ermittelung des Gehaltes an Cottonöl ermöglicht die Bestimmung der Jodzahl nach der von mir kürzlich angegebenen Methode ${ }^{1}$ ). Aus zahlreichen Versuchen ergab sich nämlich, dass $100 \mathrm{mg}$ Schweinefett $23-27 \mathrm{mg}$, $100 \mathrm{mg}$ Cottonöl $43-45 \mathrm{mg}$ Jod absorbiren. Aus der gefundenen Jodzahl lässt sich daher die Menge des Cottonöls sehr annähernd schätzen. Nimmt man als mittlere Jodzahl für Schweinefett 24, für Cottonöl 44 an, so lässt sich die Jodzahl für ein Schweinefett mit $10 \%$ Cottonöl z. B. wie folgt berechnen:

$$
\begin{aligned}
\text { Jodzahl für } 90 \text { Theile Schweinefett } & =21,6 \\
\ll \ll 10 \ll \text { Cottonöl. }= & =4,4 \\
\text { berechnete Jodzahl des Gemenges }= & =26,0
\end{aligned}
$$

In dieser Weise berechnet sich für ein Gemenge von Schweinefett mit

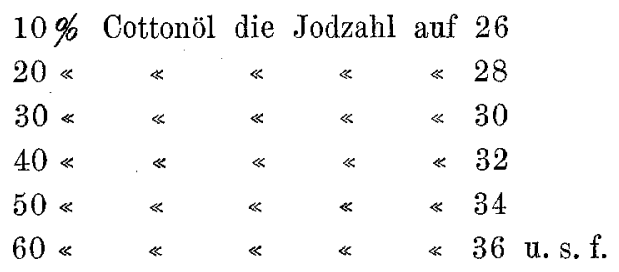

Zur experimentellen Prüfung dieser Berechnung wurden von einem

\begin{tabular}{|c|c|c|c|c|}
\hline & & & $\begin{array}{c}\text { Gefundene } \\
\text { Jodzahl }\end{array}$ & $\begin{array}{c}\text { Berechnete } \\
\text { Jodzahl }\end{array}$ \\
\hline Reines & Schweinefet & . & . 25,0 & - \\
\hline Gemenge & e mit $10 \%$ & Cottonöl & 26,0 & 26 \\
\hline$\ll$ & $\ll 20 \ll$ & $\ll$ & 27,5 & 28 \\
\hline * & $\ll 30 \ll$ & $\ll$ & 30,0 & 30 \\
\hline$\ll$ & $\ll \quad 40 \ll$ & $\ll$ & 31,8 & 32 \\
\hline
\end{tabular}
Schweinefett Gemenge mit 10,20,30 und $40 \%$ Cottonöl hergestellt und darin die Jodzahl bestimmt. Dabei ergab:

Diese Zahlen zeigen, dass die Jodzahl entsprechend dem Gehalt an Cottonöl zunimmt.

Die gefundenen Jodzahlen können nun der Natur der Sache nach nicht zu einer genauen Berechnung des Cottonölgehaltes dienen, sie

1) Diese Zeitschrift 32, 181. 
geben aber doch die Grundlage zu einer sehr annähernden Schätzung desselben. Man wird z. B. wohl mit Sicherheit annehmen können, dass ein Schweinefett mit der Jodzahl $2810-20 \%$, ein solches mit der Jodzahl $3020-30 \%$ Cottonöl enthält und so weiter. Zweifel kann man aber haben, wenn die Jodzahlen 26-28 gefunden werden, da einerseits reines Schweinefett mit der Jodzahl 27, andererseits Cottonöl enthaltendes Schweinefett mit der Jodzahl 26 vorkommen kann. In solchen Zweifelsfällen gibt aber die von mir oben beschriebene Schwefelsäureprobe sichere Entscheidung. Hat man die Zahl 26-27,5 gefunden und bei der Schwefelsäureprobe keine deutliche Dunkelfärbung erhalten, so enthielt das Schweinefett sicher kein Baumwollsamenöl, trat aber eine deutliche Dunkelfärbung ein, so ist man berechtigt, anzunehmen, dass das Schweinefett etwa 10\% Cottonöl enthält.

Auf Grund dieser Ausführungen kann man an reines Schweinefett folgende Anforderungen stellen:

1. Es darf sich bei der Schwefelsäureprobe nur strohgelb, höchstens rothgelb färben.

2. Es darf keine höhere Jodzahl als 27 ergeben.

Bei der Untersuchung ist stets die Bestimmung der Jodzahl und zugleich die Schwefelsäureprobe vorzunehmen, um den sicheren Nachweis des Cottonöls zu führen.

Als Beleg für die Beziehung zwischen Jodzahl und Schwefelsäureprobe füge ich das Ergebniss der Untersuchung einer grösseren Reihe von Schweinefettproben bei, welche zum Theil direct von Grosshandlungen stammen, zum Theil aber, so wie sie an das Publikum verkauft werden, von Kleinhändlern und Metzgern entnommen sind. (Siehe Tabelle Seite 308.)

Die Zahlen der nachstehenden Tabelle zeigen, dass diejenigen Proben, welche eine hohe Jodzahl ergaben, bei der Schwefelsäureprobe sich auch braun färbten und daher mit Sicherheit als cottonölhaltig zu betrachten sind. Eine Ausnahme macht nur die Probe Nr. 15 mit der hohen Jodzahl 32, welche nach der Schwefelsäureprobe als frei von Cottonöl zu betrachten ist. Es ist sehr leicht möglich, dass diese Probe mit einem anderen Oele vermischt ist; Sicherheit hierïber wird die ermittelte Jodzahl aber erst geben, wenn durch eine grössere Anzahl von Versuchsreihen die mittlere Jodzahl für reines Schweinefett mit grösserer Sicherheit, als dies bis jetzt der Fall ist, festgestellt sein wird. 
308 Gantter: Nachweis ron Baumwollsamenöl in Schweinefett etc.

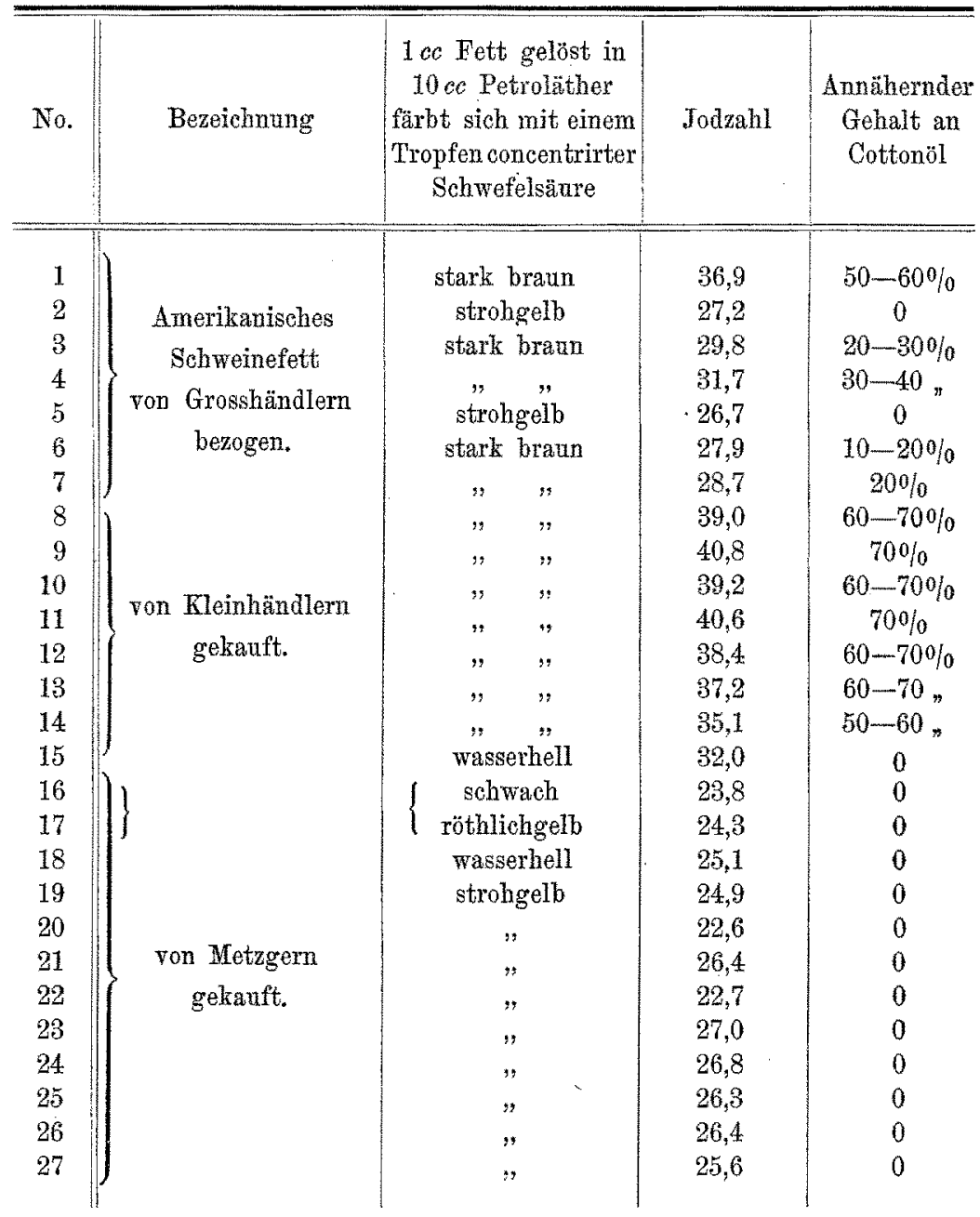

Heilbronn, Laboratorium des Verfassers, Januar 1893. 